\title{
Temporal Analysis of Sentiment Events - A Visual Realization and Tracking
}

\author{
Dipankar Das ${ }^{1}$, Anup Kumar Kolya ${ }^{1}$, Asif Ekbal ${ }^{2}$ and Sivaji Bandyopadhyay ${ }^{1}$ \\ ${ }^{1}$ Department of Computer Science and Engineering, Jadavpur University, \\ Kolkata 700 032, India \\ ${ }^{2}$ Department of Information Engineering and Computer Science, University of Trento, Italy \\ \{dipankar.dipnil2005, anup.kolya, asif.ekbal\}@gmail.com, sivaji_cse_ju@yahoo.com
}

\begin{abstract}
In recent years, extraction of temporal relations for events that express sentiments has drawn great attention of the Natural Language Processing (NLP) research communities. In this work, we propose a method that involves the association and contribution of sentiments in determining the event-event relations from texts. Firstly, we employ a machine learning approach based on Conditional Random Field (CRF) for solving the problem of Task C (identification of event-event relations) of TempEval-2007 within TimeML framework by considering sentiment as a feature of an event. Incorporating sentiment property, our system achieves the performance that is better than all the participated state-of-the-art systems of TempEval 2007. Evaluation results on the Task C test set yield the F-score values of 57.2\% under the strict evaluation scheme and $58.6 \%$ under the relaxed evaluation scheme. The positive or negative coarse grained sentiments as well as the Ekman's six basic universal emotions (or, fine grained sentiments) are assigned to the events. Thereafter, we analyze the temporal relations between events in order to track the sentiment events. Representation of the temporal relations in a graph format shows the shallow visual realization path for tracking the sentiments over events. Manual evaluation of temporal relations of sentiment events identified in 20 documents sounds satisfactory from the purview of event-sentiment tracking.
\end{abstract}

Keywords: Temporal Relations, CRF, TempEval-2007, TimeML, Sentiment Event, Visual Tracking.

\section{Introduction}

The kinds of states which change and thus might need to be located in time are referred as events in the present context. The event entities are represented by finite clauses, nonfinite clauses, nominalizations, event-referring nouns, adjectives and even some kinds of adverbial clauses. In general, the events are described in different newspaper texts, stories and other important documents where occurrence time of events, temporal location and ordering of the events are specified. Several earlier methods have been proposed for detecting and tracking events from text archives [1]. 
On the other hand, text does not only contain informative contents, but also some attitudinal private information, including emotional states. Human emotion described in texts is an important cue for our daily communication too. But, the identification of emotional states from texts is not an easy task as emotion is not open to any objective observation or verification [2]. Nowadays, in the Natural Language Processing (NLP) communities, several research activities on sentiment and/or emotion analysis are in full swing. Sentiment of people is important as it has great influence on our society. Our main motivation to investigate the insides of event-sentiment relation lies with the facts that though events and sentiments are closely coupled with each other from social, psychological and commercial perspectives, there has been very little attention regarding their detection The identification of the temporal relations between two events by taking the sentiment feature into account is also crucial to analyze and track human sentiments. This is also important in a wide range of other NLP applications that include temporal question answering, document summarization, current information retrieval systems etc.

Mishne and de Rijke [3] proposed a system, MoodViews ${ }^{1}$ to analyze the temporal change of sentiment. MoodViews analyzes multiple sentiments by using 132 sentiments used in LiveJournal ${ }^{2}$. Although our concept for the sentiment graph is similar to MoodViews, we focus on temporal relations between events associated with similar or different types of sentiments. With respect to information visualization, Havre et al. proposed a system called ThemeRiver [4] that visualizes thematic flows along with timeline. Although our approach is different from ThemeRiver, we focus on visualization of sentiment flows on events based on temporal expressions. The temporal sentiment identification from social events has been carried out in [5]. In their task, the authors have analyzed the temporal trends of sentiments and topics from a text archive that has timestamps in Weblog and news articles and produces two kinds of graphs, topic graph that shows temporal change of topics associated with a sentiment and sentiment graph that shows temporal change of sentiments associated with a topic. In contrast, our present task incorporates the knowledge of temporal relations (e.g. AFTER, BEFORE, OVERLAP) instead of timestamps for temporal sentiment tracking. In addition to that, we also analyze the role of sentiment in identifying temporal relations between the events.

Let us consider the following example:

"The prime minister of India told Friday that he has talked with top commander of Indian military force and sent a team to recover the host of Taj Hotel hijacked."

For example, in the above sentence, the native speakers can quickly identify the ordering of the three events, namely 'hijacking', 'talking' and 'sending' as: hijacking $\rightarrow$ talking $\rightarrow$ sending even though the temporal relations such as 'before', 'after' or 'overlap' never appeared in the text. But, the above example also shows the presence of underlying sentiments (as shown in underlined script) scribed in the sentence. The TempEval-2007 challenge addressed the question of identifying temporal relations by using a common corpus on which research systems competed to find temporal relations [6]. Our present aim is not only to identify the temporal relations from the events but also to identify the sentiments associated with the

\footnotetext{
${ }^{1} \mathrm{http}: / /$ moodviews.com/

${ }^{2}$ http://www.livejournal.com/
} 
events, to determine the contribution of sentiment in identifying temporal relations as well as to track the sentiments over the events based on temporal relations.

The present system identifies temporal relations between the events by considering the contribution of the sentiment property into account. We propose a machine learning approach based on Conditional Random Field (CRF) [7] for solving the problem of Task C (identification of event-event relations) of TempEval-2007 within TimeML framework. The task is to identify the temporal relations between the events that occur in two consecutive sentences and to classify the event pairs into their respective temporal classes. Incorporating sentiment property into the set of other standard features, the proposed system outperforms all the participated state-ofthe-art systems of TempEval 2007 with the F-score values of 56.87\% under the strict evaluation scheme and $59.20 \%$ under the relaxed evaluation scheme. The positive or negative coarse-grained sentiments as well as the Ekman's [8] six basic universal emotions or fine-grained sentiments (happiness, sadness, anger, fear, surprise and disgust) are assigned to the events. Based on the temporal relations, the events from each of the documents are represented using a graph that shows the shallow path for identifying the sentiment changes over events. Manual evaluation of temporal relations of sentiment events identified in 20 documents sounds satisfactory from the purview of event-sentiment tracking.

The rest of the paper is organized as follows. Section 2 describes the sentiment based temporal relation identification using CRF. The evaluation schemes and results are discussed in Section 3. The tagging of the events with sentiments, generation and tracking of the event-sentiment relational graph and the evaluation of the eventsentiment tracking system along with the associated results are discussed in Section 4. Finally Section 5 concludes the paper.

\section{CRF based System for Identifying Temporal Relations}

The Task $\mathrm{C}$ at TempEval-2007 was involved with the automatic identification of temporal relations holding between verb events in adjacent sentences. There are two types of events such as, main-event and sub-ordinate event. The main-event is determined from multiple sentences by following very shallow, syntactic-based criteria within the scope of a sentence. It has been observed that syntactically subordinate events are dominated by main-event in coordination relations and the main-event is the first one in the sentence string. The events expressions were annotated in the source in accordance with the TimeML standard [9]. For all the tasks, data were provided for training and testing that includes annotations identifying: (1) sentence boundaries, (2) all temporal referring expression as specified by TIMEX3, (3) all events as specified in TimeML and (4) selected instances of temporal relations, as relevant to the given task. For task $\mathrm{C}$, a restricted set of event terms, whose stems occurred twenty times or more in the TimeBank corpus, was identified. This set is referred to as the Event Target List or ETL. Furthermore, only the event expressions that occur within the ETL are considered. In the training and test data, TLINK annotations for these temporal relations are provided. The only difference being that in the test data the relation type is withheld. 


\subsection{Pair-Wise Classification using CRF}

In the present approach, the identifications and classifications of temporal relations are based on a supervised machine learning algorithm, Conditional Random Field (CRF) that can include arbitrary set of features and still can avoid over fitting in a principled manner. We consider the temporal relation identification task as a pairwise classification problem in which the EVENT target pairs are modelled. In the present task, we report only Task $\mathrm{C}$ that identifies temporal relations between the events that appear in two adjacent sentences. The events and temporal expressions (TE) were annotated in the source in accordance with the TimeML standard [9]. The set of temporal relations to be predicted includes: OVERLAP, BEFORE, AFTER, BEFORE-OR-OVERLAP, OVERLAP-OR-AFTER and VAGUE.

The main advantage of CRF is the assumption of conditional independence of the observed data. In generative approach, this might be too restrictive for a considerable number of object classes. Unlike ME, CRF does not suffer from the label bias problem. CRF can include arbitrary set of features and has the ability of automatic feature induction.

\subsection{Features}

We use the gold-standard TimeBank features for training and testing the CRF model. The features are extracted automatically from the respective datasets but we mainly use the various combinations of the following features:

(i). Event Class: This is denoted by the 'EVENT' tag and used to annotate those elements in a text that mark the semantic events described by it.

(ii). Event Stem: This feature extracts the stem of the head event.

(iii). Event and Time Strings: This feature is used to denote the actual event strings and time.

(iv). Part of Speech (POS) of Event Terms: POS information is very useful to identify the even-event relations. The features values may be either of ADJECTIVE, NOUN, VERB, and PREP.

(v). Event Tense: This feature is useful to capture the standard distinctions among the grammatical categories of verbal phrases. The tense attribute can have values, namely PRESENT, PAST, FUTURE, INFINITIVE, PRESPART, PASTPART, or NONE

(vi). Event Aspect: It denotes the aspect of the events. The aspect attribute may take values, PROGRESSIVE, PERFECTIVE and PERFECTIVE PROGRESSIVE or NONE

(vii). Event Polarity: Polarity of an event instance is represented by the boolean value, POSITIVE or NEGATIVE.

(viii). Event Modality: The modality attribute is only present if there is a modal word that modifies the instance.

(ix). Type of Temporal Expression: It represents the temporal relationship holding between events, times or between an event and a time of the event.

(x). Temporal Signal: This is used to represent the temporal prepositions. 
(xi).Temporal Relation between the Document Creation Time and Temporal Expression in the Target Sentence: The value of this feature could be "greater than", "less than", "equal", or "none".

\section{Incorporating Sentiment Feature}

The sentiment is an important cue that effectively describes the events associated with it. The binary classification of the sentiments as well as the fine-grained categorization of Ekman's six emotions is utilized to qualify the event properties. The sentiment attributes are identified for each of the sentences. Here, we mainly employ the word to sentence level emotion tagging module [11] for identifying the sentiment properties of events. The features for positive and negative sentiments as well as a representative feature with respect to all the six emotions are assigned for classifying the event pairs into the temporal classes.

The sentiment or emotional verbs play an important role in identifying the temporal relations. To accomplish the goal, we include a special feature for sentiment verbs that are identified using SentiWordNet [12] or WordNet Affect lists [14] or VerbNet [15]. The verbs of SentiWordNet or WordNet Affect are identified using the Part-of-Speech (POS) information. On the other hand, VerbNet associates the semantics of a verb with its syntactic frames and combines traditional lexical semantic information such as thematic roles and semantic predicates, with syntactic frames and selectional restrictions. Verb entries in the same VerbNet class share common syntactic frames, and thus they are believed to have the same syntactic behavior. The VerbNet files containing the verbs with their possible subcategorization frames and membership information are stored in XML file format. For example, the emotional verbs "love" and "enjoy" are members of the admire-31.2-1 class and "enjoy" also belongs to the class want-32.1-1. The XML files of VerbNet are preprocessed to build up a general list that contains all member verbs and their available syntax information retrieved from VerbNet. The main criterion that is considered for selecting the frames is the presence of "emotional_state" type predicate associated with the frame semantics.

We obtain the training and testing datasets from the TempEval-2007 evaluation task. The datasets are preprocessed for the specified CRF format. Thereafter, we extract features in the form of vectors from the annotated training data. The feature vectors consisting of the available features for each <main-event, main-event> and $<$ main-event, next subordinate event, previous subordinate event, main-event> pair in the TimeBank corpus are identified. Now, we have a training data in the form ( $\mathrm{Wi}$, $T i$ ), where, $W i$ is the $i^{\text {th }}$ pair along with its feature vector and $T i$ is its corresponding TempEval relation class. All the feature vectors are extracted from the training data. The temporal relations are annotated by one of the labels, such as BEFORE, BEFORE-OR-OVERLAP, OVERLAP, OVERLAP-OR-AFTER, AFTER or VAGUE. We have trained CRF using the automatically extracted feature vectors and by defining the appropriate feature template. The models are created from the training set and the feature template. The same feature extraction methodology is again repeated for the test data. An unknown instance <main-event, main-event $>$ or $<$ mainevent, next subordinate event, previous subordinate event, main-event $>$ is assigned the appropriate output label, i.e., OVERLAP, BEFORE, AFTER, BEFORE- 
OR-OVERLAP, OVERLAP-OR-AFTER and VAGUE, depending upon the probabilities, learned in the CRF model. The output label predicted by the CRF is matched against the reference label.

\section{Evaluation of Temporal Event Identification System}

For TempEval -2007, the tasks were defined in such a way that a simple pair-wise comparison is possible since it was not required to create a full temporal graph and judgments are made in isolation. There are three basic temporal relations (BEFORE, OVERLAP, and AFTER) as well as three disjunctions over this set (BEFORE-OROVERLAP, OVERLAP-OR-AFTER and VAGUE). The organizers used two scoring schemes: strict and relaxed.

The strict scoring scheme only counts exact matches as success. For example, if the key is OVERLAP and the response is BEFORE-OR-OVERLAP then this is counted as 'failure'. The standard definitions of precision and recall are followed: Precision $=R_{c} / R$ and Recall $=R_{c} / K$, where, $R c$ is the number of correct answers in the response, $R$ is the total number of answers in the response and $K$ is the total number of answers in the key. For the relaxed scoring scheme, precision and recall are defined as Precision $=R c w / R$ and Recall $=R c w / K$, where, $R c w$ reflects the weighted number of correct answers. The $F$-score is measured as follows where $\operatorname{Pr}=$ Precision and Re $=$ Recall: $F$-score $=2^{*} P r^{*} R e /(P r+R e)$. We have developed a number of CRF models based on the features and/or feature templates included into it. We have a training data in the form $(W i, T i)$, where, $W i$ is the $i^{\text {th }}$ pair along with its feature vector and $T i$ is its corresponding TempEval relation class. Models are built based on the training data and the feature template. During evaluation, we obtain the highest performance for the following feature templates as shown in Figure 1. In the figure, $\mathrm{w}_{\mathrm{i}}$ : Current <event, event> pair, $\mathrm{w}_{(\mathrm{i}-\mathrm{n})}$ : Previous $\mathrm{n}^{\text {th }}<$ <event, event> pair, $\mathrm{W}_{(\mathrm{i}+\mathrm{n})}:$ Next $\mathrm{n}^{\text {th }}<$ event, event> pair, $t_{i-1}$ previous <event, event> pair.

\begin{tabular}{|c|}
\hline $\mathrm{W}_{(\mathrm{i}-3)}$ \\
\hline $\mathrm{W}_{(\mathrm{i}-2)}$ \\
\hline $\mathrm{W}_{(\mathrm{i}-1)}$ \\
\hline $\mathrm{w}_{\mathrm{i}}$ \\
\hline $\mathrm{W}_{\mathrm{i}+1}$ \\
\hline $\mathrm{W}_{(\mathrm{i}+2)}$ \\
\hline $\mathrm{w}_{(\mathrm{i}+3)}$ \\
\hline Combination of $w_{i-1}$ and $w_{i}$ \\
\hline Combination of $w_{i}$ and $w_{i+1}$ \\
\hline Dynamic output tag $\left(t_{i-1}\right)$ of the previous pair \\
\hline Feature vector of $w_{i}$ of other features \\
\hline
\end{tabular}

Fig. 1. Best Feature Template of the CRF based System for <main-event, next sub-event, prev sub-event, main-event $>$ relation 
The test data consists of 20 articles from TimeBank [8]. The performance is assessed with three evaluation metrics, namely precision $(\mathrm{P})$, recall $(\mathrm{R})$ and F-score (FS). The systems are evaluated in terms of two scoring schemes, 'strict' and 'relaxed'. The strict scoring scheme counts only exact matches, while the relaxed one gives credit to partial semantic matches too. Evaluation results [13] show that the system performs better with the context size of seven (i.e., previous three, current and the next three <main-event, next-subordinate-event, previous- subordinate-event, main-event> pairs), tense, aspect and temporal class features. It shows the precision, recall and F-score values of $43.8 \% 43.8 \%$ and $43.8 \%$ respectively under the strict evaluation scheme and $46.9,46.9 \%$ and $46.9 \%$, respectively under the relaxed evaluation scheme. For the sub-ordinate event, the system demonstrated the precision, recall and F-score values of $55.1 \%, 55.1 \%$ and $55.1 \%$, respectively for the strict evaluation scheme and $56.9 \%, 56.9 \%$ and $56.9 \%$, respectively for the relaxed evaluation scheme. Table 1 shows the results by incorporating the sentiment feature into the system. For the main-event, sentiment feature in CRF based system performs better with the margins of 1.4 percentage F-scores in the strict evaluation scheme and 1.5 percentage F-scores in the relaxed evaluation scheme.

Table 1. Precision (P), Recall (R) and F-scores of CRF based system

\begin{tabular}{lccccccc}
\hline Techniques & & Strict & \multicolumn{5}{c}{ Relaxed } \\
& P & $\mathbf{R}$ & FS & P & R & FS \\
CRF (main event) & 0.438 & 0.438 & 0.438 & 0.469 & 0.469 & 0.469 \\
CRF (main event) +Sentiment & 0.452 & 0.452 & 0.452 & 0.484 & 0.484 & 0.484 \\
Feature & & & & & & \\
CRF (subordinate event) & 0.551 & 0.551 & 0.551 & 0.569 & 0.569 & 0.569 \\
CRF (subordinate event) & 0.572 & 0.572 & 0.572 & 0.586 & 0.586 & 0.586 \\
+Sentiment Feature & & & & & & \\
\hline
\end{tabular}

The incorporation of sentiment feature also shows that the CRF is most effective to handle the subordinate-event in association with the knowledge regarding the sentiment property of the events. It shows the overall performance improvement of 2.1 and 1.7 percentage points over the earlier model in the strict and relaxed evaluation scheme, respectively. The system also exhibits superior performance for the subordinate-event over the main-event.

\section{Event-Sentiment Tagging}

Opinion or Emotion or Sentiment analysis is a recent sub discipline at the crossroads of information retrieval [16] and computational linguistics [17]. Information is concerned not only with the subject or topic or event of a document but also with the sentiment or emotion it expresses. It has a rich set of applications such as tracking users' sentiments about products or events or about political candidates as expressed in online forums, customer relationship management, stock market prediction, social networking etc. Not only the classification of reviews [18] or newspaper articles [19] 
or blogs [20] but the Question-Answering systems [21] and current Information Retrieval systems [22] are also increasingly incorporating sentiment analysis within their scopes.

In the present task, we use the TimeML corpus for assigning coarse grained sentiments such as positive or negative and fine grained sentiments such as Ekman's six emotions (happiness, sadness, anger, fear, surprise and disgust) at the sentence level. Event-sentiment relational graph for each of the documents is generated based on the temporal relations identified using a CRF based supervised event-event relation identification system. The generation of the event-sentiment relational graph using an open source graphical system helps to visualize and track the changes of sentiments between consecutive and remote events of a document. The sentiment change between two consecutive events is termed as sentiment twist and change between rare or remote events containing one or more intermediate events is termed as sentiment transition. The manual evaluation of the event-sentiment system sounds satisfactory.

\subsection{Tagging of Sentiments to Events}

The investigation mainly focused on analyzing the impacts of coarse grained and fine grained sentiments on events that are present in the TempEval-2007 TimeML corpus. Ekman's [8] six basic emotion types, such as happiness, sadness, anger, fear, surprise, and disgust are considered as fine-grained sentiment whereas two different valences, positive and negative are considered as coarse grained and are assigned to the sentences that contain events. Other sentences are considered as neutral. The sentiment tagging systems [10] [11] work at two levels, word level followed by sentence level.

The CRF based machine learning approach that incorporates several singleton features (e.g. POS, words from SentiWordNet [12], question words, reduplication, punctuation markers or special symbols (!, @, \$, ?)) as well as different combination of context features (e.g., unigram, bigram etc.) are employed for word level emotion tagging. The system [10] demonstrates F-score of 72.27\% for English SemEval 2007 affect sensing news corpus containing 2,500 development word tokens. Incorporation of error analysis and equal distribution of emotion tags with the non-emotion tag improves the word level emotion tagging and an overall F-score of $83.65 \%$ is achieved by the system on 1,500 test word tokens of the news corpus.

The sentential emotions and valence tags are assigned based on the word level emotion tagged constituents. The system calculates six different emotion tag weights from SentiWordNet and applies the tag weights on the word level emotion tagged data to acquire sentence level emotion scores for each emotion type. A sentence level emotion tag that has the maximum emotion score is assigned to each sentence. The system shows the F-scores of 66.66\%, 59.33\%, 62.32\%, 62.70\%, 65.89\% and 62.67\% for happy sad, anger, disgust, fear and surprise emotion classes, respectively on 200 test sentences. On the other hand, the polarity information of the emotion tag weights helps in calculating the valence score for each of the sentences. The total emotion tag weights acquired from the different emotion tags in a sentence are treated as the valence or coarse grained sentiments (positive and negative) of the sentence. It has 
been observed that the system achieves an average F-score of $66.41 \%$ for coarse grained sentiment tagging on 250 test sentences [11].

As there is no emotion-annotated information available in TempEval-2007 corpus, each test sentence of the corpus was annotated with single emotion tag and evaluated by us successively. The systems [10] [11] have been applied on 20 test articles of the TempEval event corpus. Manual evaluation shows that the system achieves an average F-score of $64.23 \%$ for emotion tagging with respect to all emotion classes. But, the sentences of the TempEval corpus is annotated with positive and negative sentiments and the evaluation yields an average F-score of $66.23 \%$.

\subsection{Generation of Event-Sentiment Relational Graph}

The Ekman's six different emotions along with positive, negative or neutral sentiments are tagged with the sentences containing one or more potential events. If we consider the positive, negative and neutral valences as coarse grained sentiment events and Ekman's six emotions as the fine-grained sentiments, by hypothesis, the temporal relations also exist among the sentiment events and the relations between each of the events are represented using a directed graph. The temporal relations between each of the successive sentiment or neutral events have already been identified by the CRF based system as described in Section 2.

An open source graphical tool ${ }^{3}$ has been used to represent the temporal relations among the events. The tool uses an XML file schema to store the information regarding nodes as well as the edges of a graph (as shown in Figure 2). For each of the documents of TempEval-2007 event corpus, a separate graph is generated. The sentiment of each sentence is assigned to its containing event and each event is represented using a graphical node. The event nodes that are of similar sentiments are connected to their corresponding sentiment hubs based on their annotated sentential sentiment tags.

\subsection{Tracking of Event-Sentiment Relational Graph}

The tracking of sentiments includes sentiment twist and sentiment transition. The sentiment twist between two consecutive events and sentiment transition among more than two events are identified by arriving at the corresponding sentiment hub. The sentiment change or tracking of sentiments between two consecutive events or sentiment twist is identified from the AFTER, BEFORE and OVERLAP temporal relations. The ambiguities of the OVERLAP relations are identified by the notion of BEFORE-OR-OVERLAP, OVERLAP-OR-AFTER relations. It has been observed that the maximum length of an event chain or sentiment transition in the TempEval 2007 corpus is four. The number of instances of the sentiment transitions is less than the number of instances of the sentiment twists in the TimeML corpus. Hence, the sentiment transition or tracking of sentiment is identified based on the sentiment twists of the intermediate event pairs in an event chain.

\footnotetext{
${ }^{3}$ http://www.hpl.hp.com/research/idl/projects/graphs/guess.html
} 


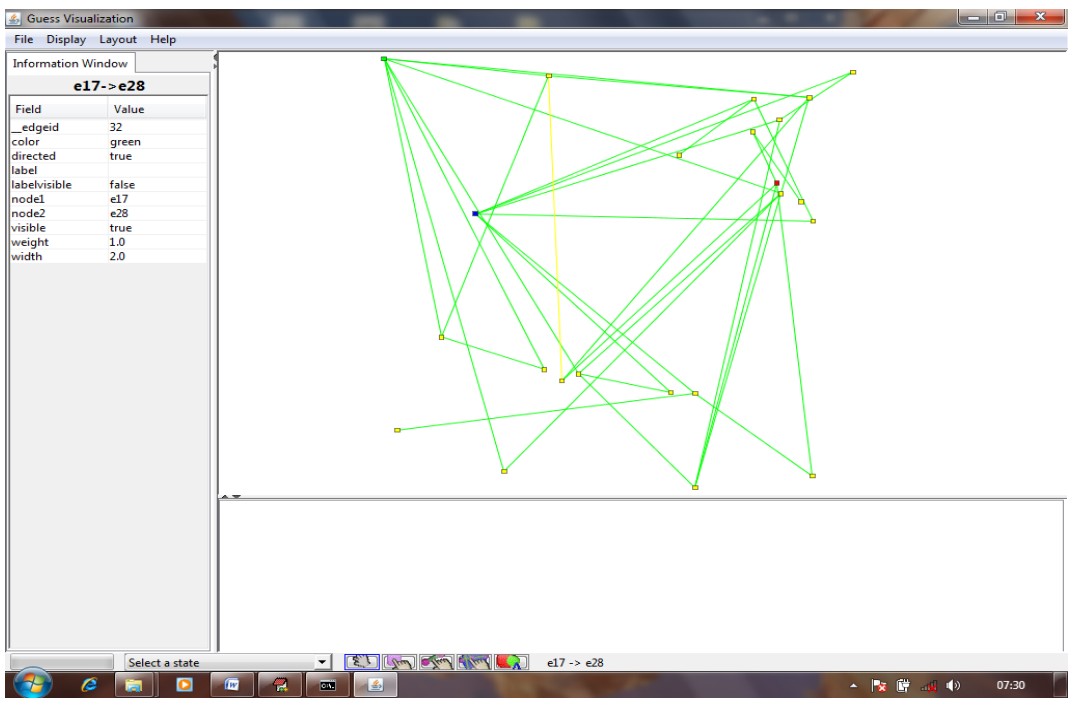

Fig. 2. Snapshot of a Sentiment-Event Directed Graph for a document

\subsection{Evaluation of Event-Sentiment Tracking}

The sentiment events are identified from 20 test documents. Apart from sentiment twist, the maximum number of participating events in a sentiment transition is $3 \sim 4$. The results of the event-sentiment tracking are shown in Table 2. In the present experiments, we have considered all the six emotions of Ekman's (happiness, sadness, anger, fear, surprise and disgust) but the results only consider the single average emotion instead of six. Results show that the performance of the system is comparatively better in case of identifying sentiment tracking between coarse grained sentiments rather than coarse to fine grained sentiments and vice versa. It has to be mentioned that though the system performs satisfactory in identifying the event sentiment tracking path, most of the errors have occurred due to the misleading characteristics of the system in assigning neutral tags to the sentences with implicit sentiments. The rest of the errors occur in detecting the immediate sentiment changes of reverse polarity (+ve/-ve) in the sentiment twists. It shows that the complementary sentiment changes in sentiment twists are not always reliable without proper reasoning but in case of sentiment transitions, the reversibility may occur by changing sentiments in the intermediate event nodes in an event chain.

\section{Conclusion}

In this paper, we have reported our work on temporal relation identification under the TempEval 2007 evaluation exercise. The Task C of TempEval-2007was involved with the identification of six relations between the events in two consecutive 
sentences. Evaluation results show that the CRF based system outperforms all the state-of-the-art participating systems by including sentiment as the feature with all other available features of the TimeBank corpus. The sentiment tagged events, their visualization and tracking as well the evaluation show a promising venue of research. In future, we would like to employ the system in identifying sentiment changes from lengthy event chains in order to investigate the potential reasoning behind the sentiment change.

Table 2. Results of F-scores of the Event-Sentiment Tracking

\begin{tabular}{lccc}
\hline $\begin{array}{l}\text { Source-Destination } \\
\text { Pair }\end{array}$ & \multicolumn{3}{c}{ Twist/Transition between \# Events } \\
& & Three & Four \\
+ve $\rightarrow$-ve & 0.7032 & 0.6924 & 0.6821 \\
+ ve $\rightarrow$ neutral & 0.7365 & -- & -- \\
+ ve $\rightarrow$ Emotion & 0.6414 & 0.6277 & 0.6087 \\
& & & \\
-ve $\rightarrow$ +ve & 0.7065 & 0.6833 & 0.6724 \\
-ve $\rightarrow$ neutral & 0.7151 & -- & -- \\
-ve $\rightarrow$ Emotion & 0.6075 & 0.5877 & 0.5729 \\
& & & \\
neutral $\rightarrow+$ ve & 0.7011 & -- & -- \\
neutral $\rightarrow$-ve & 0.6898 & -- & -- \\
neutral $\rightarrow$ Emotion & 0.6337 & -- & - \\
& & & \\
Emotion $\rightarrow$ +ve & 0.6227 & 0.6077 & 0.5802 \\
Emotion $\rightarrow$-ve & 0.6393 & 0.6207 & 0.6162 \\
Emotion $\rightarrow$ neutral & 0.6210 & -- & -- \\
\hline
\end{tabular}

Acknowledgments. The work reported in this paper was supported by a grant from the India-Japan Cooperative Programme (DSTJST) 2009 Research project entitled "Sentiment Analysis where AI meets Psychology" funded by Department of Science and Technology (DST), Government of India.

\section{References}

1. Wayne, C.L.: Multilingual Topic Detection and Tracking: Successful Research Enabled by Corpora and Evaluation. In: Language Resources and Evaluation Conference (LREC), pp.1487--1494 (2000)

2. Quirk R., Greenbaum, S., Leech G., and Svartvik, J.: A comprehensive Grammar of the English Language, Longman, New York (1985)

3. Mishne, G. and de Rijke, M.: MoodViews: Tools for Blog Mood Analysis. In: AAAI 2006 Spring Symposium on Computational Approaches to analyzing Weblogs (AAAICAAW2006) (2006)

4. Havre, S., Hetzler, E., Whitney, P., and Nowell, L.: ThemeRiver: Visualizing Thematic Changes in Large Document Collections. In: IEEE Transactions on Visualization and Computer Graphics, vol.8(1), pp. 9--20 (2002) 
5. Fukuhara, T., Nakagawa, H., Nishida, T.: Understanding Sentiment of People from News Articles: Temporal Sentiment Analysis of Social Events. In: ICWSM'2007 Boulder, Colorado, USA (2007)

6. Verhagen, M., Gaizauskas, R., Schilder, F., Hepple, M., Katz, G., Pustejovsky, and J.: SemEval-2007 Task 15: TempEval Temporal Relation Identification. In: Proceedings of the $4^{\text {th }}$ International Workshop on Semantic Evaluations (semEval-2007), pp. 75--80, Prague, June 2007. Association for Computational Linguistics (2007)

7. Lafferty, J., McCallum, A.K., Pereira, and F.: Conditional Random Fields: Probabilistic Models for Segmenting and Labeling Sequence Data. In: proceedings of $18^{\text {th }}$ International Conference on Machine Learning (2001)

8. Ekman, P.: Facial expression and emotion. American Psychologist, vol. 48(4), pp.384--392 (1993)

9. Pustejovsky, J., Castano, J., Ingria, R., Sauri, R., Gaizauskas, R., Setzer, A., Katz, G., Radev.: TimeML: Robust Specification of Event and Temporal Expressions in Text. In: Proceedings of the Fifth International Workshop on Computational Semantics (IWCS-5), Tilburg, January (2003)

10. Das, D., and Bandyopadhyay, S.: Sentence Level Emotion Tagging on Blog and News Corpora. Journal of Intelligent System (JIS), vol. 19(2), pp. 125-134 (2010)

11. Das, D., and Bandyopadhyay, S.: Sentence Level Emotion Tagging. In: proceedings of the International Conference on Affective Computing \& Intelligent Interaction DOI:10.1109/ACII.2009.5349598, pp. 375-380, Amsterdam, Netherlands (2009)

12. Esuli, A., and Sebastiani, F.: SENTIWORDNET: A Publicly Available Lexical Resource for Opinion Mining. In: LREC-06 (2006)

13. Kolya, A. K., Ekbal, A., and Bandyopadhyay S.: A Supervised Machine Learning Approach for Event Event Relation Identification. In: PACLIC 2010. November 4-7, Sendai, Japan (2010)

14. Strapparava, C., and Valitutti, A.: WordNet-Affect: an affective extension of WordNet. In: proceedings of the 4th International Conference on Language Resources and Evaluation, pp. 1083-1086 (2004)

15. Kipper-Schuler, K.: VerbNet: A broad-coverage, comprehensive verb lexicon. Ph.D. thesis, Computer and Information Science Dept., University of Pennsylvania, Philadelphia,PA (2005)

16. Sood, S., and Vasserman, L.: ESSE: Exploring Mood on the Web. In: Proceedings of the $3^{\text {rd }}$ International AAAI Conference on Weblogs and Social Media (ICWSM) Data Challenge Workshop (2009)

17. Wiebe, J., Wilson, T., Rebecca, F., Bell, M., and Martin, M.: Learning Subjective Language. Computational Linguistics, vol. 30, pp. 277--308 (2004)

18. Turney, P.D.: Thumbs Up or Thumbs Down? Semantic Orientation Applied to Unsupervised Classification of Reviews. In: Proceedings of the 40th Annual Meeting of the Association for Computational Linguistics, pp. 417-- 424 (2002)

19. Lin, K. H.-Y., Yang, C., and Chen, H.-H.: What Emotions News Articles Trigger in Their Readers? In: Proceedings of SIGIR, pp. 733--734 (2007)

20. Yang, C., Lin, K. H.-Y., and Chen, H.-H.: Emotion classification Using Web Blog Corpora. In: IEEE, WIC, ACM International Conference on Web Intelligence, pp. 275--278 (2007)

21. Cardie, C., Wiebe, J., Wilson, T., and Litman, J.D.: Combining Low-Level and Summary Representations of Opinions for Multi-Perspective Question Answering. In: New Directions in Question Answering, pp. 20--27 (2003)

22. Pang, B., and Lee, L.: opinion mining and sentiment analysis. In: Foundations and Trends in Information Retrieval 2, vol. 1(2), pp. 1--135 (2008) 